\title{
Second moment constraints and the control problem of Markov jump linear systems
}

\author{
Alessandro N. Vargas ${ }^{1,2, *, \dagger}$, Walter Furloni ${ }^{3}$ and João B. R. do Val ${ }^{3}$ \\ ${ }^{1}$ Universidade Tecnológica Federal do Paraná, UTFPR, Av. Alberto Carazzai 1640, 86300-000 Cornelio Procópio-PR, \\ Brazil \\ ${ }^{2}$ Basque Center for Applied Mathematics, BCAM, Alameda de Mazarredo 14, E-48009 Bilbao, Vizcaya, Spain \\ (1 January-28 February 2011) \\ ${ }^{3}$ Universidade Estadual de Campinas, UNICAMP, FEEC-DT, Av. Albert Einstein 400, 13083-852 Campinas-SP, Brazil
}

\begin{abstract}
SUMMARY
This paper addresses the optimal solution for the regulator control problem of Markov jump linear systems subject to second moment constraints. We can characterize and obtain the solution explicitly using linear matrix inequalities techniques. The constraints are imposed on the second moment of both the system state and control vector, and the optimal solution is obtained in a computable form. To illustrate the usefulness of the approach, specially that for systems subject to abrupt variations and physical limitations, we present an application for one joint of the European Robotic Arm. Copyright (C) 2012 John Wiley \& Sons, Ltd.
\end{abstract}

Received 29 July 2011; Revised 14 December 2011; Accepted 3 June 2012

KEY WORDS: Markov jump systems; optimal stochastic control; constrained control; linear-quadratic problems; linear matrix inequalities

\section{INTRODUCTION}

Many practical systems are subject to abrupt changes on its modes of operation, and the class of stochastic systems known as Markov jump linear systems (MJLS) has been proved to be useful to model a large number of them. We can cite the papers [1-8] and the monograph [9] as a small sample of recent theoretical developments and applications of MJLS. However, although fairly consolidated on many of its aspects, the knowledge of MJLS regarding constraints is incipient. Indeed, to the best of the authors' knowledge, the paper [10] is unique to handle constraints for MJLS in the complete state observation setup. The conditions presented in [10] are sufficient only and a certain degree of conservatism exists. Our approach advances in this topic by introducing a method to compute the optimal solution (i.e., with no conservatism) for the linear state-feedback control problem of MJLS with second moment constraints.

Consider the following discrete-time Markovian jump system:

$$
x_{k+1}=A_{\theta_{k}} x_{k}+B_{\theta_{k}} u_{k}+H_{\theta_{k}} w_{k}, \quad \forall k \geqslant 0, x_{0} \in \mathbf{R}^{n}, \theta_{0} \in \mathscr{S},
$$

where $x_{k}, u_{k}$, and $w_{k}, k=0,1, \ldots$ are processes taking values, respectively, in $\mathbf{R}^{n}, \mathbf{R}^{m}$, and $\mathbf{R}^{q}$, which denote the system state, control, and additive noisy input. The process $\left\{\theta_{k}\right\}$ represents a discrete-time homogeneous Markov chain taking values in a finite set $\mathscr{S}=\{1, \ldots, \eta\}$, and the matrix parameters $A_{\theta_{k}}=A_{i}, B_{\theta_{k}}=B_{i}$, and $H_{\theta_{k}}=H_{i}$ are given whenever $\theta_{k}=i \in \mathscr{S}, k \geqslant 0$. The noisy input $\left\{w_{k}\right\}$ forms an independent and identically distributed process with zero mean and

\footnotetext{
*Correspondence to: Alessandro N. Vargas, UTFPR, Av. Alberto Carazzai 1640, 86300-000 Cornelio Procópio-PR, Brazil.

${ }^{\dagger}$ E-mail: avargas@utfpr.edu.br
} 
covariance matrix equal to the identity for all $k \geqslant 0$. We also assume that $\left(\Omega, \mathcal{F},\left\{\mathcal{F}_{k}\right\}, \mathcal{P}\right)$ is a fixed probability space for (1), where $\left\{\mathcal{F}_{k}\right\}$ represents the filtration, and each $\mathcal{F}_{k}, k \geqslant 0$ corresponds to the $\sigma$-field generated by $\left(x_{0}, \theta_{0}, \ldots, x_{k}, \theta_{k}\right)$. Both $x_{k}$ and $\theta_{k}$ are completely available and hence completely observed at the $k$ th stage.

To measure the performance of the system (1), we consider the usual quadratic cost of $N$ stages

$$
J_{N}\left(x_{0}, \theta_{0}\right):=\mathrm{E}_{x_{0}, \theta_{0}}\left[\sum_{k=0}^{N}\left\|C_{\theta_{k}} x_{k}+D_{\theta_{k}} u_{k}\right\|^{2}\right],
$$

where $\mathrm{E}_{x_{0}, \theta_{0}}[\cdot] \equiv \mathrm{E}\left[\cdot \mid x_{0}, \theta_{0}\right]$, and the matrices $C_{\theta_{k}}$ and $D_{\theta_{k}}, k \geqslant 0$ are known whenever $\theta_{k} \in \mathscr{S}$.

It is well known that most of the practical realizations of control systems are subject to physical limitations, typically because of the saturation of actuators, limitations on the current or voltage in the involved electronic circuits, safety on operating conditions or economical requirements, among others [11], and in the solution to the optimal control, it is important to take these constraints into consideration. In the deterministic scenario (i.e., with $\theta_{k} \equiv 1$ and $w_{k} \equiv 0$ ), the usual approach suggests hard constraints to deal with limitations on the system state and control vectors [12-14]. But this strategy is meaningless in the stochastic context because the random values may easily drive either the system state or control actions to an infeasible region [15]. A more natural and reasonable strategy in this case consists in taking constraints related to the expected value operator. This paper contributes toward this direction by introducing constraints on the second moment of both the system state $x_{k}$ and control action $u_{k}, k \geqslant 0$, as follows.

Consider $\varphi: \mathbf{R}^{n} \mapsto \mathbf{R}^{n}$ as the operator of the componentwise square vector, that is, for a given vector $z=\left[z_{[1]}, \ldots, z_{[n]}\right]^{\prime} \in \mathbf{R}^{n}$, one has that $\varphi(z)=\left[z_{[1]}^{2}, \ldots, z_{[n]}^{2}\right]^{\prime}$. Using this operator, we can impose second moment constraints on the system (1) as

$$
\mathrm{E}\left[\varphi\left(x_{k}\right)\right]<\delta_{k} \quad \text { and } \quad \mathrm{E}\left[\varphi\left(u_{k}\right)\right]<\gamma_{k}, \quad \forall k \geqslant 0,
$$

where $\left\{\delta_{k}\right\}$ and $\left\{\gamma_{k}\right\}$ denote arbitrary sequences of positive orthants from $\mathbf{R}^{n}$ and $\mathbf{R}^{q}$, respectively. Although the format of (3) allows us to impose adequate bounds for the covariance matrix of $x_{k}$ and $u_{k}$, it also allows us to set amplitude bounds for the expected value of $x_{k}$ and $u_{k}$ (see Section 3 for further details and a numerical application).

Control actions in the linear state-feedback format are vital in many practical applications, mainly due to their simple implementation and maintenance; see, for instance, the schemes of linear control implementation in [16, Chapter 4.3] based on electronic operational amplifiers and in [17] based on valves, transmitters, and other industrial devices. The easiness of synthesis and applications motivates us to constrain the control action in the usual linear state-feedback form as

$$
u_{k}=G_{\theta_{k}}(k) x_{k}, \quad \forall k \geqslant 0,
$$

where $G_{\theta_{k}}(k)$ is a gain matrix of dimension $m \times n$ to be determined for each $k \geqslant 0$. Note that the linear feedback form as in (4) in fact attains the optimum in the unconstrained control problem [4; 9, Chapter 4]. To clarify the control structure, let us assume that $G(k)=\left\{G_{1}(k), \ldots, G_{\eta}(k)\right\}$, $k=0, \ldots, N$ is a gain matrix sequence as in (4), and let $\mathscr{G}$ be the set made up by all admissible gain sequences.

The novelty of this paper is that it introduces a method to minimize the cost (2) subject to the second moment inequalities (3) and linear control synthesis (4). Namely, we present the solution for the following underlying constrained control problem:

$$
J_{N}^{*}\left(x_{0}, \theta_{0}\right):=\min _{\{G(0), \ldots, G(N)\} \in \mathscr{G}} J_{N}\left(x_{0}, \theta_{0}\right) \quad \text { s.t. (1)-(4). }
$$

The solution of the control problem proposed in (5) is given in terms of the linear matrix inequality (LMI) approach [18-20]. Namely, the control problem is rewritten in terms of an LMI convex optimization problem and this enables us to obtain the corresponding constrained optimal solution. Hence, there is no conservatism on our approach and the constrained stochastic control problem in (5) is feasible if and only if our LMI conditions are valid. 
The paper is organized as follows. Section 2 presents basic notation and concepts, and the main result. The main result, concerning the optimal solution for the constrained control problem, is presented in Theorem 2.1. Finally, in Section 3, we present an application of the derived optimal solution to control one joint of the European Robotic Arm (ERA) with constraints.

\section{BASIC CONCEPTS AND MAIN RESULT}

Let $\mathbf{R}^{r}$ denote the $r$ th dimensional Euclidean space with the usual norm $\|\cdot\|$. Let $\mathbf{M}^{r, s}$ represent the linear space formed by all $r \times s$ real matrices. Let $\mathbf{S}^{r, r}$ represent the normed linear subspace of $\mathbf{M}^{r, r}$ given by all symmetric matrices such as $\left\{U \in \mathbf{M}^{r, r}: U=U^{\prime}\right\}$, where $U^{\prime}$ denotes the transpose of $U$. Consider also $\mathbf{S}^{r 0}\left(\mathbf{S}^{r+}\right)$ its closed (open) convex cone of positive semi-definite (definite) matrices $\left\{U \in \mathbf{S}^{r, r}: U \geqslant 0(>0)\right\}$. Set $\mathscr{S}=\{1, \ldots, \eta\}$, and let $\mathbb{M}^{r, s}=\left\{U=\left(U_{1}, \ldots, U_{\eta}\right): U_{i} \in \mathbf{M}^{r, s}, i \in \mathscr{S}\right\}$. The identity element of $\mathbb{M}^{r, r}$ is denoted by $\mathbb{I}$, that is, $V \mathbb{I}=\mathbb{I} V=V$ whenever $V \in \mathbb{M}^{r, r}$. We denote by $\mathbb{S}^{r 0}$ ( $\mathbb{S}^{r+}$ ) the set made up by $U_{i} \in \mathbf{S}^{r 0}$ $\left(U_{i} \in \mathbf{S}^{r+}\right)$ for all $i \in \mathscr{S}$. Given $U, V \in \mathbb{M}^{r, r}$, we employ the ordering $U>V(U \geqslant V)$ meaning that $U_{i}-V_{i}$ is positive definite (semi-definite) for each $i \in \mathscr{S}$ and similarly for other mathematical relations. Recalling the Schur complement lemma [18, p. 7], given $U \in \mathbb{M}^{r, n}, V \in \mathbb{S}^{r+}$, and $S \in \mathbb{S}^{n+}$, we have

$$
V>U S^{-1} U^{\prime} \Leftrightarrow\left[\begin{array}{cc}
V & U \\
U^{\prime} & S
\end{array}\right]>0 .
$$

Consider $\operatorname{tr}\{\cdot\}$ as the trace operator. Define the inner product on the space $\mathbb{M}^{r, s}$ as

$$
\langle U, V\rangle=\sum_{i=1}^{\eta} \operatorname{tr}\left\{U_{i}^{\prime} V_{i}\right\}, \quad \forall V, U \in \mathbb{M}^{r, s} .
$$

We denote by $e_{i}, i=1, \ldots, r$, the vector with 1 in the $i$ th coordinate and 0 elsewhere, thus $\left[e_{1}|\ldots| e_{r}\right]$ retrieves the identity matrix. In addition, we define $\psi: \mathbb{S}^{r 0} \mapsto \mathbf{R}^{r}$ as the operator

$$
\psi(U)=\left[\begin{array}{c}
e_{1}^{\prime}\left(\sum_{i=1}^{\eta} U_{i}\right) e_{1} \\
\vdots \\
e_{r}^{\prime}\left(\sum_{i=1}^{\eta} U_{i}\right) e_{r}
\end{array}\right], \quad \forall U \in \mathbb{S}^{r 0} .
$$

The transition probability matrix is denoted by $\mathbb{P}=\left[p_{i j}\right]$ for all $i, j \in \mathscr{S}$. The state of the Markov chain at a certain time $k$ is determined according to an associated probability distribution $\pi(k)$ on $\mathscr{S}$, namely, $\pi_{i}(k):=\operatorname{Pr}\left(\theta_{k}=i\right)$.

We define the operator $\mathscr{T}=\left\{\mathscr{T}_{1}, \ldots, \mathscr{T}_{\eta}\right\}: \mathbb{S}^{n 0} \mapsto \mathbb{S}^{n 0}$, as

$$
\mathscr{T}_{i}(U)=\sum_{j=1}^{\eta} p_{j i} U_{j}, \quad i=1, \ldots, \eta, \quad \forall U \in \mathbb{S}^{r 0} .
$$

Let us define the conditional second moment matrix of the system state $x_{k}, k \geqslant 0$ as

$$
X_{i}(k)=\mathrm{E}\left[x_{k} x_{k}^{\prime} \mathbb{1}_{\left\{\theta_{k}=i\right\}}\right], \quad \forall i \in \mathscr{S},
$$

where $\mathbb{1}_{\{\cdot\}}$ stands for the Dirac measure. Setting $X(k)=\left\{X_{1}(k), \ldots, X_{\eta}(k)\right\} \in \mathbb{S}^{n 0}, k \geqslant 0$, we are able to express an equivalent form of evaluating (7) and the corresponding cost (2).

Proposition 2.1

[9, Chapter 3] There holds

$$
J_{N}\left(x_{0}, \theta_{0}\right)=\sum_{k=0}^{N}\left\langle(C+D G(k))^{\prime}(C+D G(k)), X(k)\right\rangle,
$$


where $X(k) \in \mathbb{S}^{n 0}$ satisfies the recurrence

$$
X(k+1)=\mathscr{T}\left((A+B G(k)) X(k)(A+B G(k))^{\prime}+\pi(k) H H^{\prime}\right), \quad \forall k \geqslant 0,
$$

with $X_{i}(0)=x_{0} x_{0}^{\prime} \mathbb{1}_{\left\{\theta_{0}=i\right\}}$ for each $i \in \mathscr{S}$.

The result of Proposition 2.1 provides a convenient way to compute the cost $J_{N}\left(x_{0}, \theta_{0}\right)$, yet with no constraints. To expand upon the control problem with constraints, let us first show that $\mathrm{E}\left[\varphi\left(x_{k}\right)\right]=\psi(X(k))$ for each $k \geqslant 0$. Indeed, from the definition of $\varphi(\cdot)$, we have

$$
\varphi\left(x_{k}\right)=\left[\begin{array}{c}
\left(e_{1}^{\prime} x_{k}\right)^{2} \\
\vdots \\
\left(e_{n}^{\prime} x_{k}\right)^{2}
\end{array}\right]=\left[\begin{array}{c}
e_{1}^{\prime} x_{k} x_{k}^{\prime} e_{1} \\
\vdots \\
e_{n}^{\prime} x_{k} x_{k}^{\prime} e_{n}
\end{array}\right] \in \mathbf{R}^{n}, \quad \forall k \geqslant 0
$$

This enables us to write

$$
\mathrm{E}\left[\varphi\left(x_{k}\right)\right]=\sum_{i=1}^{\eta} \mathrm{E}\left[\varphi\left(x_{k}\right) \mathbb{1}_{\theta_{k}=i}\right]=\left[\begin{array}{c}
e_{1}^{\prime}\left(\sum_{i=1}^{\eta} \mathrm{E}\left[x_{k} x_{k}^{\prime} \mathbb{1}_{\theta_{k}=i}\right]\right) e_{1} \\
\vdots \\
e_{n}^{\prime}\left(\sum_{i=1}^{\eta} \mathrm{E}\left[x_{k} x_{k}^{\prime} \mathbb{1}_{\theta_{k}=i}\right]\right) e_{n}
\end{array}\right]=\psi(X(k)), \quad \forall k \geqslant 0,
$$

which shows the claim. A similar reasoning, combined with (4), leads to the other identity

$$
\mathrm{E}\left[\varphi\left(u_{k}\right)\right]=\psi\left(G(k) X(k) G(k)^{\prime}\right), \quad \forall k \geqslant 0 .
$$

The next result is a straightforward consequence of these identities.

Lemma 2.1

The constraints in (3) hold if and only if

$$
\psi(X(k))<\delta_{k} \quad \text { and } \quad \psi\left(G(k) X(k) G(k)^{\prime}\right)<\gamma_{k} \quad \text { for each } k \geqslant 0 .
$$

\section{Remark 2.1}

In view of Proposition 2.1 and Lemma 2.1, the control problem of finding the minimum $J_{N}^{*}\left(x_{0}, \theta_{0}\right)$ as stated in (5) can be recasted as that of finding a gain sequence $G(k), k=0, \ldots, N$ that minimizes (8) subject to (9) and (10). The optimization problem in the setting of (8)-(10) is nonlinear with respect to $G(k), k=0, \ldots, N$, and this represents a barrier toward the solution. To overcome this difficulty, we provide in the sequel a form of expressing that nonlinear problem as a convenient LMI one.

\subsection{Preliminary results for the LMI representation}

Given an admissible gain sequence $G(k)=\left\{G_{1}(k), \ldots, G_{\eta}(k)\right\} \in \mathbb{M}^{m, n}, k \geqslant 0$, we denote the corresponding closed loop matrices by

$$
A(k)=A+B G(k) \quad \text { and } \quad C(k)=C+D G(k), \quad \forall k \geqslant 0 .
$$

The proof of the next result is available in Appendix.

Lemma 2.2

The constraints (8)-(10) are feasible if and only if for each sufficiently small constant $\epsilon>0$, there corresponds a matrix sequence $P(k)=P^{\epsilon}(k) \in \mathbb{S}^{n 0}, k \geqslant 0$, such that

$$
J_{N}\left(x_{0}, \theta_{0}\right) \leqslant \sum_{k=0}^{N}\left\langle C(k)^{\prime} C(k), P(k)\right\rangle \leqslant J_{N}\left(x_{0}, \theta_{0}\right)+\epsilon
$$

where

$$
\mathscr{T}\left(A(k) P(k) A(k)^{\prime}+\pi(k) H H^{\prime}\right)-P(k+1)<0, \quad P(0)=X(0),
$$




$$
\psi(P(k))<\delta_{k}, \text { and } \psi\left(G(k) P(k) G(k)^{\prime}\right)<\gamma_{k}, \quad \forall k \geqslant 0 .
$$

Let us now consider the following optimization problem with $L \in \mathbb{S}^{n+}, R(k) \in \mathbb{S}^{n+}, W(k) \in$ $\mathbb{S}^{n+}$, and $V(k) \in \mathbb{S}^{n+}, k \geqslant 0$ as parameter variables.

$$
\begin{aligned}
& \rho:=\inf \sum_{k=0}^{N}\langle W(k), \mathbb{I}\rangle \\
& {\left[\begin{array}{cc}
W(k) & C(k) \mathscr{T}(R(k-1)) \\
\star & \mathscr{T}(R(k-1))
\end{array}\right]>0, \quad k=1, \ldots, N ;} \\
& {\left[\begin{array}{cc}
W(0) & C(0) L \\
\star & L
\end{array}\right]>0} \\
& {\left[\begin{array}{cc}
R(k)-\pi(k) H H^{\prime} & A(k) \mathscr{T}(R(k-1)) \\
\star & \mathscr{T}(R(k-1))
\end{array}\right]>0, \quad k=1, \ldots, N-1 ;} \\
& {\left[\begin{array}{cc}
R(0)-\pi(0) H H^{\prime} & A(0) L \\
\star & L
\end{array}\right]>0 ; \quad L-X(0)>0 ;} \\
& \psi(\mathscr{T}(R(k-1)))<\delta_{k}, \quad k=1, \ldots, N-1, \quad \psi(L)<\delta_{0} ; \\
& {\left[\begin{array}{cc}
V(k) & G(k) \mathscr{T}(R(k-1)) \\
\star & \mathscr{T}(R(k-1))
\end{array}\right]>0, \quad \psi(V(k))<\gamma_{k}, \quad k=1, \ldots, N-1 ;} \\
& {\left[\begin{array}{cc}
V(0) & G(0) L \\
\star & L
\end{array}\right]>0 \quad \text { with } \quad \psi(V(0))<\gamma_{0}}
\end{aligned}
$$

The symbol $\star$ represents the symmetric of block $(1,2)$.

\section{Lemma 2.3}

There holds $\rho=J_{N}\left(x_{0}, \theta_{0}\right)$.

\section{Proof}

The proof is divided into two parts.

Part 1: [We show that (11)-(13) $\Rightarrow(14)-(21)$ and that $\rho \leqslant J^{N}\left(x_{0}, \theta_{0}\right)$.]

With (11)-(13) being valid, then for a given $\epsilon>0$, there exist $P(k)=P^{\epsilon}(k), k=0, \ldots, N$, and sufficiently small positive constants $\xi_{0}, \xi_{1}, \ldots, \xi_{N-1}$, such that

$$
\mathscr{T}_{i}\left(A(k) P(k) A(k)^{\prime}+\pi(k) H H^{\prime}\right)-P_{i}(k+1)<-\mathscr{T}_{i}\left(\xi_{k} \mathbb{I}\right)<0, \quad \forall i \in \mathscr{S}, k=0, \ldots, N-1 .
$$

But if we set

$$
R_{i}(k)=A_{i}(k) P_{i}(k) A_{i}(k)^{\prime}+\pi_{i}(k) H_{i} H_{i}^{\prime}+\xi_{k} I,
$$

then we obtain $\mathscr{T}_{i}(R(k))<P_{i}(k+1)$. Hence,

$$
\begin{aligned}
R_{i}(k)-\pi_{i}(k) H_{i} H_{i}^{\prime} & >A_{i}(k) P_{i}(k) A_{i}(k)^{\prime} \\
& >A_{i}(k) \mathscr{T}_{i}(R(k-1)) A_{i}(k)^{\prime}, \quad k=1, \ldots, N-1 .
\end{aligned}
$$

Applying the Schur complement lemma in (23), we obtain the inequality in (17) for each $k=1, \ldots, N$, and taking $P(0)=X(0)$ in (23), we obtain (18). 
Before showing (19)-(21), note first that $\psi(\cdot)$ is a linear operator so that we can write

$$
\psi(P(k))>\psi(\mathscr{T}(R(k-1))), \quad k=1, \ldots, N-1 .
$$

Thus, the left-hand side inequality of (13) implies (19). If, in addition, we consider a slack variable $V(k) \in \mathbb{S}^{n+}, k \geqslant 0$, such that

$$
\psi(V(k))<\gamma_{k} \quad \text { and } \quad V(k)>G(k) P(k) G(k)^{\prime}, \quad \forall k \geqslant 0,
$$

then the Schur lemma guarantees both (20) and (21). Let us now choose a sufficiently small number $\xi>0$ to define

$$
W^{\xi}(k)=C(k) P(k) C(k)^{\prime}+\xi I, \quad k=0, \ldots, N .
$$

Note from the inequality $P(k)>\mathscr{T}(R(k-1))$ that

$$
W^{\xi}(k)>C(k) \mathscr{T}(R(k-1)) C(k)^{\prime}, \quad k=1, \ldots, N .
$$

These inequalities imply (15), and (16) also follows because $P(0)=X(0)$.

Now, we show that (11) implies that $\rho \leqslant J^{N}\left(x_{0}, \theta_{0}\right)$. Indeed, we have from (11) and (24) that

$$
\epsilon+J^{N}\left(x_{0}, \theta_{0}\right) \geqslant \sum_{k=0}^{N}\left\langle C(k)^{\prime} C(k), P(k)\right\rangle=\sum_{k=0}^{N}\left\langle W^{\xi}(k), \mathbb{I}\right\rangle-(N+1) \xi .
$$

With $\rho \geqslant 0$ as in (14)-(21) and observing that

$$
\lim _{\xi \rightarrow 0} \sum_{k=0}^{N}\left\langle W^{\xi}(k), \mathbb{I}\right\rangle=\inf \sum_{k=0}^{N}\langle W(k), \mathbb{I}\rangle=\rho,
$$

we can conclude from (25) that $\epsilon+J^{N}\left(x_{0}, \theta_{0}\right) \geqslant \rho$, and taking $\epsilon \downarrow 0$, we have $J^{N}\left(x_{0}, \theta_{0}\right) \geqslant \rho$. This argument completes the proof of the Part 1 .

Part 2: [We show that (14)-(21) $\Rightarrow(11)-(13)$ and that $\rho \geqslant J^{N}\left(x_{0}, \theta_{0}\right)$ ].

Combining (17) and the Schur lemma, we have

$$
R_{i}(k)>A_{i}(k) \mathscr{T}_{i}(R(k-1)) A_{i}(k)^{\prime}+\pi_{i}(k) H_{i} H_{i}^{\prime}, \quad k=1, \ldots, N-1, \quad \forall i \in \mathscr{S} .
$$

Set $P(k)=\mathscr{T}(R(k-1))$ for each $k=1, \ldots, N$. Now, we can apply the linear operator $\mathscr{T}(\cdot)$ on both sides of (26) to obtain

$$
P(k+1)=\mathscr{T}(R(k))>\mathscr{T}\left(A P(k) A^{\prime}+\pi(k) H H^{\prime}\right), \quad k=1, \ldots, N-1,
$$

which shows (12) for $k=1, \ldots, N-1$. If one sets $P(0)=X(0)$ in (18), then one obtains (12) for $k=0$.

From (15), we have

$$
W(k)>C(k) \mathscr{T}(R(k-1)) C(k)^{\prime}=C(k) P(k) C(k)^{\prime}, \quad k=1, \ldots, N,
$$

and from (16), we have $W(0)>C(0) P(0) C(0)^{\prime}$. These inequalities and the Schur lemma applied in (19)-(21) suffice to (13). Note, in addition, that

$$
\rho=\sum_{k=0}^{N}\langle W(k), \mathbb{I}\rangle \geqslant \sum_{k=0}^{N}\left\langle C(k)^{\prime} C(k), P(k)\right\rangle .
$$

Let $X(k), k \geqslant 0$, be as in (9). Because $P(k)$ is an upper bound for $X(k)$, we have

$$
\sum_{k=0}^{N}\left\langle C(k)^{\prime} C(k), P(k) \geqslant \sum_{k=0}^{N}\left\langle C(k)^{\prime} C(k), X(k)\right\rangle=J^{N}\left(x_{0}, \theta_{0}\right),\right.
$$

which implies that $\rho \geqslant J^{N}\left(x_{0}, \theta_{0}\right)$. This argument completes the proof. 


\subsection{LMI formulation and the main result}

Our development at this point allows us to establish the LMI convex formulation that assuredly computes the minimum $J_{N}^{*}\left(x_{0}, \theta_{0}\right)$ as in (5). Firstly, note that the gain sequence $G(k), k=0, \ldots, N$ was taken fixed into the equations (14)-(21). If $G(k)$ is taken to be a variable, then we obtain a nonlinear formulation from (14)-(21), and this fact poses an important drawback, mainly for the numerical viewpoint. An alternative LMI derivation can be constructed to overcome this difficulty, as follows. Let us apply into (14)-(21) the change of variables [18, p. 193]

$$
Z(k)=G(k) \mathscr{T}(R(k-1)), \quad k=1, \ldots, N, \quad \text { and } \quad Z(0)=G(0) L .
$$

Note that this change of variables produces the following LMI optimization problem on the matrix variables $L \in \mathbb{S}^{n+}, R(k) \in \mathbb{S}^{n+}, W(k) \in \mathbb{S}^{q+}, V(k) \in \mathbb{S}^{n+}$, and $Z(k) \in \mathbb{M}^{m, n}, k \geqslant 0$.

$$
\begin{aligned}
& \rho^{*}=\inf \sum_{k=0}^{N}\langle W(k), \mathbb{I}\rangle \\
& {\left[\begin{array}{cc}
W(k) & C \mathscr{T}(R(k-1))+D Z(k) \\
\star & \mathscr{T}(R(k-1))
\end{array}\right]>0, \quad k=1, \ldots, N ;} \\
& {\left[\begin{array}{cc}
W(0) & C L+D Z(0) \\
\star & L
\end{array}\right]>0} \\
& {\left[\begin{array}{cc}
R(k)-\pi(k) H H^{\prime} & A \mathscr{T}(R(k-1))+B Z(k) \\
\star & \mathscr{T}(R(k-1))
\end{array}\right]>0, \quad k=1, \ldots, N-1 ;} \\
& {\left[\begin{array}{cc}
R(0)-\pi(0) H H^{\prime} & A L+B Z(0) \\
\star & L
\end{array}\right]>0 ; \quad L-X(0)>0 ;} \\
& \psi(\mathscr{T}(R(k-1)))<\delta_{k}, \quad k=1, \ldots, N-1, \quad \psi(L)<\delta_{0} ; \\
& {\left[\begin{array}{cc}
V(k) & Z(k) \\
\star & \mathscr{T}(R(k-1))
\end{array}\right]>0, \quad \psi(V(k))<\gamma_{k}, \quad k=1, \ldots, N-1 ;} \\
& {\left[\begin{array}{cc}
V(0) & Z(0) \\
\star & L
\end{array}\right]>0 \text { with } \psi(V(0))<\gamma_{0} .}
\end{aligned}
$$

The next result is an immediate consequence of Lemma 2.3 linked with (29).

\section{Theorem 2.1}

The LMI problem (30)-(37) is such that $\rho^{*}=J_{N}^{*}\left(x_{0}, \theta_{0}\right)$. Moreover, the control action as in (4) is optimal for the problem in (5) provided that

$$
G(k)=Z(k) \mathscr{T}(R(k-1))^{-1}, \quad k=1, \ldots, N-1, \quad \text { and } \quad G(0)=Z(0) L^{-1} .
$$

\section{Remark 2.2}

The LMI formulation in (30)-(37) provides the optimal solution for the constrained control problem posed in (5). To illustrate this result, an application for one joint of the ERA is addressed in the next section. 


\section{APPLICATION}

In this section, the usefulness of the result in Theorem 2.1 is demonstrated by means of an application for the model of one joint of the ERA, see [21-23].

The ERA system, represented by the block diagram of Figure 1, is a continuous-time system subject to failures that impose abrupt changes in the system dynamics [22,23]. These failures affect the parameter values of both the constant motor torque $F_{K_{t}}$ and the input inertial axis $F_{I_{m}}$, and these variations are assumed here to be driven by an homogeneous Markov chain with a given probability matrix, according to the values of Table I. Associating these values with the continuous-time ERA system of [22] and employing a zero-order hold with sampling period of $0.05 \mathrm{~ms}$, we obtain the discrete-time Markov jump system

$$
x_{k+1}=\left[\begin{array}{cccc}
1 & 0.05 & a_{1}^{i} & a_{2}^{i} \\
0 & 1 & a_{3}^{i} & a_{4}^{i} \\
0 & 0 & a_{5}^{i} & a_{6}^{i} \\
0 & 0 & a_{7}^{i} & a_{8}^{i}
\end{array}\right] x_{k}+\left[\begin{array}{c}
b_{1}^{i} \\
b_{2}^{i} \\
b_{3}^{i} \\
b_{4}^{i}
\end{array}\right] u_{k}+0.1 w_{k}, \quad \theta_{k}=i \in \mathscr{S}, k \geqslant 0,
$$

with $\mathscr{S}:=\{1, \ldots, 6\}$, where the values $a_{1}^{i}, \ldots, a_{8}^{i}, b_{1}^{i}, \ldots, b_{4}^{i}$ are shown in Table II. Taking

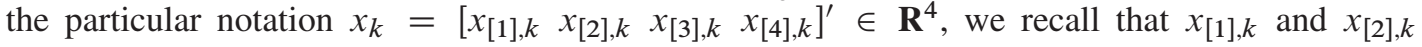

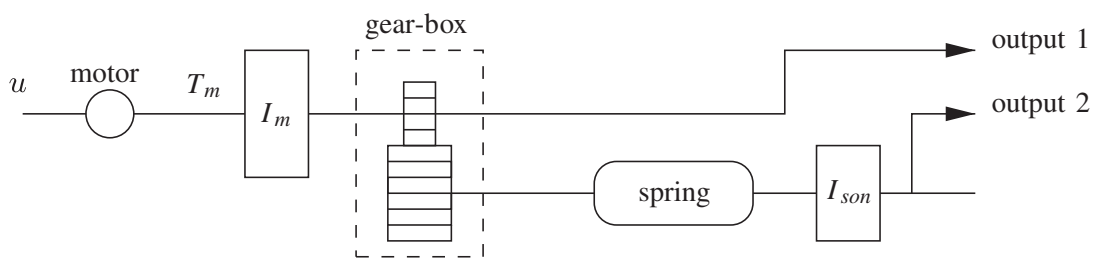

Figure 1. Block diagram of one joint of the European Robotic Arm.

Table I. Jump parameters for the motor torque and input inertial axis for the ERA system, according to the application of Section 3.

\begin{tabular}{lcccccc}
\hline Parameters & $i=1$ & $i=2$ & $i=3$ & $i=4$ & $i=5$ & $i=6$ \\
\hline$F_{K_{t}}(i)$ & 1 & 1 & 1.2 & 1.2 & 0.12 & 0.12 \\
$F_{I_{m}}(i)$ & 1 & 0.5 & 1 & 0.5 & 1 & 0.5 \\
\hline
\end{tabular}

Table II. Parameter values of the discrete-time Markov jump system in (38), which represents one joint of the European Robotic Arm system subject to failures, in accordance with the application of Section 3.

\begin{tabular}{lcccccr}
\hline Parameters & $i=1$ & $i=2$ & $i=3$ & $i=4$ & $i=5$ & $i=6$ \\
\hline$a_{1}^{i}$ & 1.3864 & 1.8277 & 1.3864 & 1.8277 & 1.3864 & 1.8277 \\
$a_{2}^{i}$ & 0.028 & 0.0449 & 0.028 & 0.0449 & 0.028 & 0.0449 \\
$a_{3}^{i}$ & 29.2528 & 3.2249 & 29.2528 & 3.2249 & 29.2528 & 3.2249 \\
$a_{4}^{i}$ & 1.3864 & 1.8277 & 1.3864 & 1.8277 & 1.3864 & 1.8277 \\
$a_{5}^{i}$ & -0.6453 & -0.9984 & -0.6453 & -0.9984 & -0.6453 & -0.9984 \\
$a_{6}^{i}$ & 0.0168 & 0.0009 & 0.0168 & 0.0009 & 0.0168 & 0.0009 \\
$a_{7}^{i}$ & -34.7158 & -3.5259 & -34.7158 & -3.5259 & -34.7158 & -3.5259 \\
$a_{8}^{i}$ & -0.6453 & -0.9984 & -0.6453 & -0.9984 & -0.6453 & -0.9984 \\
$b_{1}^{i}$ & -0.0009 & -0.0012 & -0.0011 & -0.0015 & -0.0001 & -0.0001 \\
$b_{2}^{i}$ & -0.0231 & -0.0107 & -0.0277 & -0.0129 & -0.0028 & -0.0013 \\
$b_{3}^{i}$ & 0.0008 & 0.0011 & -0.0046 & 0.001 & 0.0001 & 0.0001 \\
$b_{4}^{i}$ & 0.0176 & 0.0019 & 0.0211 & 0.0023 & 0.0021 & 0.0002 \\
\hline
\end{tabular}


$\left(x_{[3], k}\right.$ and $\left.x_{[4], k}\right)$ represent the angle position and angular velocity of the internal (output) axis of the ERA system, respectively, and the control variable $u_{k} \in \mathbf{R}$ denotes the electric current that flows into the terminal of the motor. Measurement imprecisions from their sensors are represented by the additive noise $w_{k} \in \mathbf{R}^{4}$. The information on both the system state $x_{k}$ and the Markov state $\theta_{k}$ is available for every $k \geqslant 0$, and this allows us to determine the linear state-feedback control with constraints as follows.

It is known that the maximal electric power consumed by the motor must be constrained to a certain value due to safety reasons, so that we assume the amplitude of the expected value of the electric power to not exceed $100 \mathrm{~W}$, that is, $\mathrm{E}\left[\varrho_{k}\right]<100$, where the electric power applied to the motor is $\varrho_{k}=u_{k}^{2} r, k \geqslant 0$. Assuming that the internal resistance of the motor is $r=1 \Omega$, we have

$$
\mathrm{E}\left[\varrho_{k}\right]=\mathrm{E}\left[u_{k}^{2}\right]=\mathrm{E}\left[\varphi\left(u_{k}\right)\right]<100, \quad \forall k \geqslant 0 .
$$

Note that the inequality in (39) assures that the electric power limit is obeyed. In addition, let us set

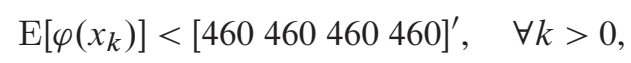

with $x_{0}=\left[\begin{array}{llll}0.8808 & 1.0902 & -0.4943 & 1.4825\end{array}\right]^{\prime}$. We also assume that

$$
C_{i}^{\prime} \equiv\left[\begin{array}{cccccc}
1 & 0 & 0 & 0 & 0 & 0 \\
0 & 1 & 0 & 0 & 0 & 0 \\
0 & 0 & 1 & 0 & 0 & 0 \\
0 & 0 & 0 & 1 & 0 & 0
\end{array}\right], \quad D_{i}^{\prime} \equiv\left[\begin{array}{cccccc}
0 & 0 & 0 & 0 & 1 & 0 \\
0 & 0 & 0 & 0 & 0 & 1
\end{array}\right], \quad \forall i \in \mathscr{S},
$$

and that the homogeneous Markov chain is driven by

$$
\mathbb{P}=\left[\begin{array}{llllll}
0.9 & 0.07 & 0.03 & 0 & 0 & 0 \\
0.7 & 0.1 & 0.05 & 0.15 & 0 & 0 \\
0.85 & 0.05 & 0.1 & 0 & 0 & 0 \\
0.6 & 0.05 & 0.05 & 0.25 & 0.05 & 0 \\
0.6 & 0 & 0 & 0 & 0.35 & 0.05 \\
0.5 & 0 & 0 & 0.05 & 0.15 & 0.3
\end{array}\right] \text {, and } \pi(0)=\left[\begin{array}{l}
1 \\
0 \\
0 \\
0 \\
0 \\
0
\end{array}\right] .
$$

Theorem 2.1 is evaluated with $N=30$ for both the unconstrained and constrained control problems. Although both solutions respect the bounds proposed in (40), the safety limit of the electric power consumed by the motor is clearly violated in the unconstrained case according to Figure 2.
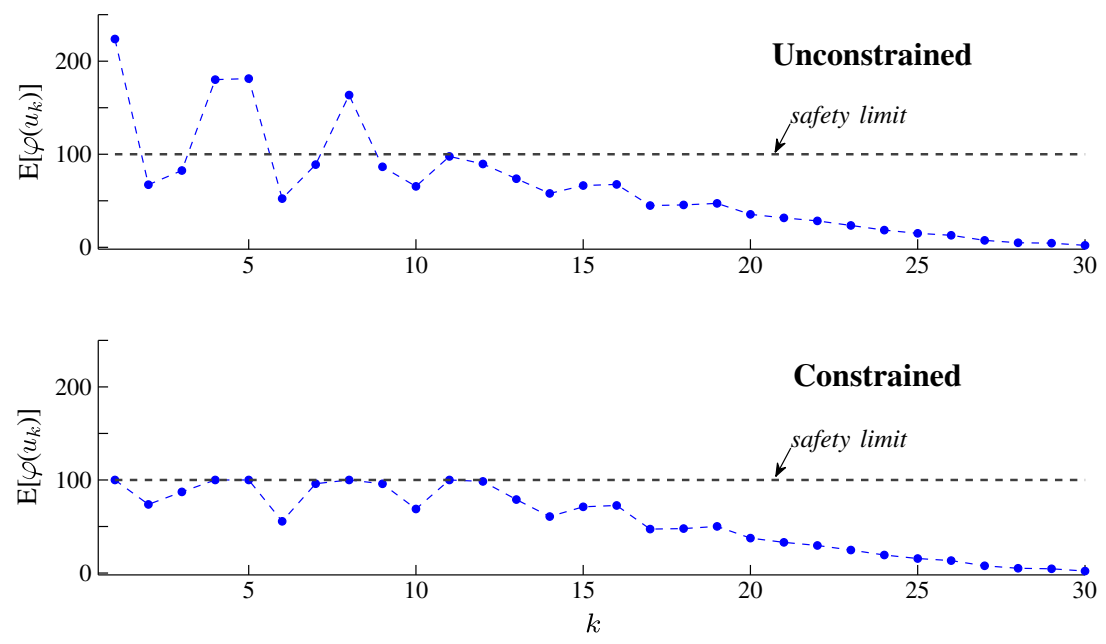

Figure 2. Second moment trajectory of the control $\mathrm{E}\left[\varphi\left(u_{k}\right)\right], k \geqslant 0$, for the European Robotic Arm (ERA) system according to the application of Section 3 . The picture indicates the electric power consumed by the ERA motor and is subject to a safety limit, which is violated in the unconstrained case. 

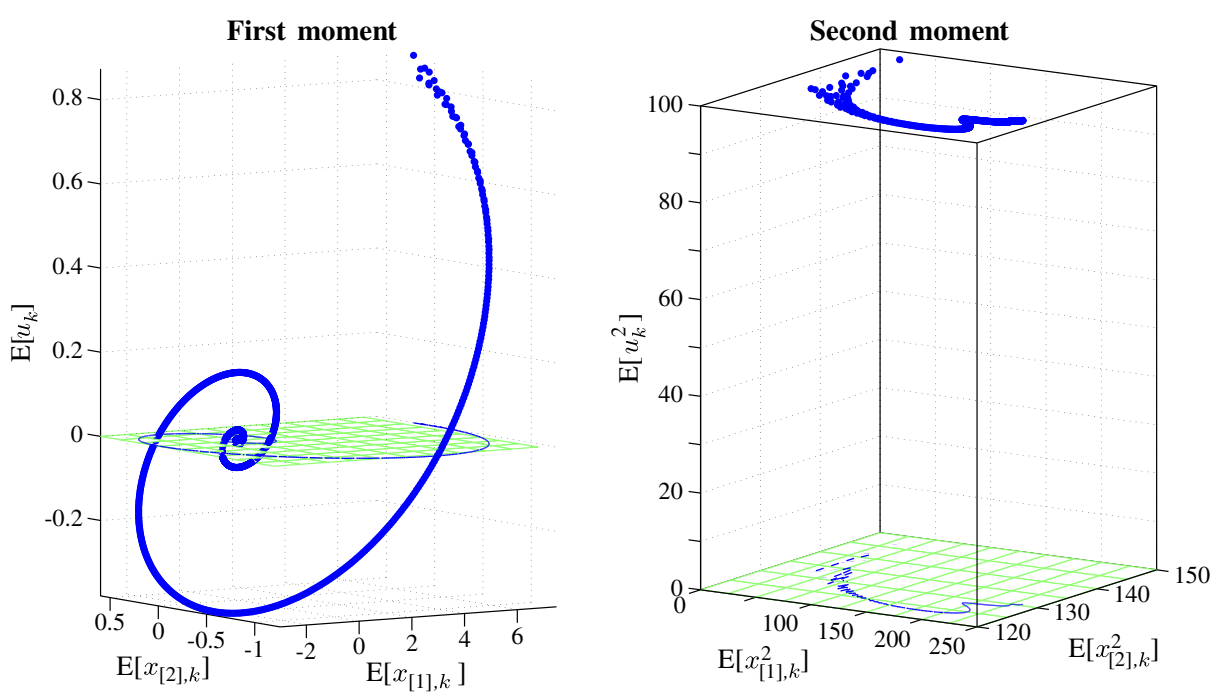

Figure 3. The curves represent the first and second moment values for the angle position $x_{[1], k}$, the angular velocity $x_{[2], k}$, and the motor electric current $u_{k}$ of the European Robotic Arm (ERA) system. The quantity $\mathrm{E}\left[u_{k}^{2}\right]$ denotes the electric power consumed by the ERA motor, which obeys the safety limit of $100 \mathrm{~W}$, according to the application of Section 3.

This fact reinforces the importance of the approach of Theorem 2.1, which is able to provide the optimal solution while maintaining the physical constraints under a desired level.

The optimal constrained cost, obtained from the corresponding numerical evaluation, is

$$
\rho^{*}=J_{N}^{*}\left(x_{0}, \theta_{0}\right)=1.6982 \times 10^{4} .
$$

In order to clarify the behavior of the ERA system under a long-term plan, we associate the receding horizon control strategy with the constrained system (38)-(40) (see $[4,15,24]$, and the references therein for further details on receding horizon control). The corresponding numerical evaluation for both the first and second moments of the angle position and angular velocity of the internal axis, and electric current, is depicted in Figure 3. Note in the figure that $\mathrm{E}\left[x_{[\ell], k}\right] \rightarrow 0$ (as $k \rightarrow \infty$ ) and $\sup _{k \geqslant 0} \mathrm{E}\left[x_{[\ell], k}^{2}\right]<\infty, \ell=1,2$. In fact, this holds for $\ell=1,2,3,4$. This evidence allows us to conclude that the ERA system (38) is not only asymptotically stable in the mean (i.e., $\mathrm{E}\left[x_{k}\right] \rightarrow 0$ as $k \rightarrow \infty$ ) but also second moment stable (i.e., $\sup _{k \geqslant 0} \mathrm{E}\left[\varphi\left(x_{k}\right)\right]<\infty$ ) (see [25] for a discussion on these stochastic stability concepts).

\section{APPENDIX A}

\section{Proof of Lemma 2.2}

The goal here is to show that (8)-(10) suffice to (11)-(13). The contrary implication, that is, (11)-(13) suffice to (8)-(10), is immediate if one takes $\epsilon \rightarrow 0$ into Lemma 2.2.

Let us assume that (8)-(10) hold. For the sake of notational simplicity, set $\Sigma(k)=\mathscr{T}\left(\pi(k) H H^{\prime}\right)$, for each $k \geqslant 0$, and

$$
\mathscr{T}(k, U):=\mathscr{T}\left((A+B G(k)) U(A+B G(k))^{\prime}\right), \quad \forall U \in \mathbb{S}^{n 0}, \forall k \geqslant 0 .
$$

Thus (9) is identical to

$$
X(k+1)=\mathscr{T}(k, X(k))+\Sigma(k), \quad \forall k \geqslant 0 .
$$

Take $P(0)=X(0)$. Given $\epsilon>0$, define $P^{\epsilon}(1)=X(1)+\epsilon \mathbb{I}$. Hence,

$$
P^{\epsilon}(1)>X(1)=\mathscr{T}(0, P(0))+\Sigma(0),
$$


which shows (12) for $k=0$. Now setting $k=1$ in (A.1), we have

$$
X(2)+\mathscr{T}(1, \epsilon \mathbb{I})=\mathscr{T}(1, X(1)+\epsilon \mathbb{I})+\Sigma(1)=\mathscr{T}\left(1, P^{\epsilon}(1)\right)+\Sigma(1) .
$$

But if we let $P^{\epsilon}(2)=X(2)+\mathscr{T}(1, \epsilon \mathbb{I})+\epsilon \mathbb{I}$, then we obtain from (A.2) that

$$
P^{\epsilon}(2)>\mathscr{T}\left(1, P^{\epsilon}(1)\right)+\Sigma(1)
$$

which shows (12) for $k=1$. Proceeding similarly with

$$
P^{\epsilon}(k)=X(k)+\sum_{j=1}^{k-1} \mathscr{T}(j, \epsilon \mathbb{I})+\epsilon \mathbb{I}, \quad k=2, \ldots, N,
$$

one can show (12) for every $k=2, \ldots, N$. Moreover, to show (13), it suffices to rescale $\epsilon>0$, if necessary, to set $P^{\epsilon}(k), k=1, \ldots, N$, to satisfy the inequalities

$$
\psi(X(k))<\psi\left(P^{\epsilon}(k)\right)<\delta_{k} \quad \text { and } \quad \psi\left(G(k) X(k) G(k)^{\prime}\right)<\psi\left(G(k) P^{\epsilon}(k) G(k)^{\prime}\right)<\gamma_{k},
$$

when $k \geqslant 0$.

Finally, by considering that the $N$ th horizon is finite, we can set an arbitrary $\bar{\epsilon}>0$ by adjusting $\epsilon>0$ in the form

$$
\bar{\epsilon}=\sum_{k=0}^{N} \sum_{j=1}^{k-1}\left\langle C(k)^{\prime} C(k), \mathscr{T}(j, \epsilon \mathbb{I})+\epsilon \mathbb{I}\right\rangle .
$$

An algebraic evaluation gives

$$
\sum_{k=0}^{N}\left\langle C(k)^{\prime} C(k), P^{\epsilon}(k)\right\rangle-\sum_{k=0}^{N}\left\langle C(k)^{\prime} C(k), X(k)\right\rangle=\sum_{k=0}^{N} \sum_{j=1}^{k-1}\left\langle C(k)^{\prime} C(k), \mathscr{T}(j, \epsilon \mathbb{I})+\epsilon \mathbb{I}\right\rangle,
$$

which implies that

$$
\sum_{k=0}^{N}\left\langle C(k)^{\prime} C(k), P^{\epsilon}(k)\right\rangle=J_{N}^{*}\left(x_{0}, \theta_{0}\right)+\bar{\epsilon} .
$$

This argument completes the proof of Lemma 2.2.

\section{ACKNOWLEDGEMENTS}

The authors acknowledge the Brazilian agencies FAPESP (FAPESP 03/06736-7) and CNPq (CNPq 71557/2009-9 and 304856/2007-0), and Spanish agency Fundación Carolina Programa 'Movilidad de profesores e investigadores Brasil-España C.2010' for financial support.

\section{REFERENCES}

1. Costa EF, do Val JBR. On the detectability and observability of continuous-time Markov jump linear systems. SIAM Journal on Control and Optimization 2002; 41(4):1295-1314.

2. Costa EF, do V, J B R, Fragoso MD. A new approach to detectability of discrete-time Markov jump linear systems. SIAM Journal on Control and Optimization 2005; 43(6):2132-2156.

3. Costa EF, Vargas AN, do V, J B R. Quadratic costs and second moments of jump linear systems with general Markov chain. Mathematics of Control, Signals, and Systems 2011; 23(1):141-157.

4. do Val JBR, Başar T. Receding horizon control of jump linear systems and a macroeconomic policy problem. Journal of Economic Dynamics and Control 1999; 23:1099-1131.

5. do Val JBR, Geromel JC, Gonçalves AP. The $\mathrm{H}_{2}$ control for jump linear systems: cluster observations of the Markov state. Automatica 2002; 38:343-349.

6. Dragan V, Morozan T. Exponential stability in mean square for a general class of discrete-time linear stochastic systems. Stochastic Analysis and Applications 2008; 26(3):495-525. 
7. Oliveira RCLF, Vargas AN, do Val JBR, Peres PLD. Robust stability, H2 analysis and stabilisation of discretetime Markov jump linear systems with uncertain probability matrix. International Journal of Control 2009; 82(3):470-481.

8. Siqueira AAG, Terra MH. A fault tolerant manipulator robot based on $\mathrm{H} 2, \mathrm{H}$-infinity, and mixed $\mathrm{H} 2 / \mathrm{H}$-infinity Markovian controls. IEEE/ASME Transactions on Mechatronics 2009; 14(2):257-263.

9. Costa OLV, Fragoso MD, Marques RP. Discrete-Time Markovian Jump Linear Systems. Springer-Verlag: New York, 2005.

10. Costa OLV, Assumpção EO, Boukas EK, Marques RP. Constrained quadratic state feedback control for discrete-time Markovian jump linear systems. Automatica 1999; 35(4):617-626.

11. Glattfelder AH, Schaufelberger W. Control Systems with Input and Output Constraints. Springer, 2003.

12. Camacho EF, Bordons C. Model Predictive Control, 2nd edn. Springer-Verlag: London, UK, 2004.

13. Mayne DQ, Rao CV, Rawlings JB, Scokaert POM. Constrained model predictive control: stability and optimality. Automatica 2000; 36:789-814.

14. Mosca E. Optimal, Predictive and Adaptive Control. Prentice Hall: Upper Saddle River, New Jersey, USA, 1994.

15. Primbs JA, Sung CH. Stochastic receding horizon control of constrained linear systems with state and control multiplicative noise. IEEE Transactions on Automatic Control 2009; 54(2):221-230.

16. Golnaraghi F, Kuo BC. Automatic Control Systems, 9th edn. John Wiley \& Sons: 111 River Street Hoboken NJ, 07030, USA, 2009.

17. Smith CA, Corripio AB. Principles and Practices of Automatic Process Control, 3rd edn. John Wiley \& Sons: 111 River Street Hoboken NJ, 07030, USA, 2005.

18. Boyd S, El Ghaoui L, Feron E, Balakrishnan V. Linear Matrix Inequalities in System and Control Theory. SIAM, Philadelphia, PA, USAQ6: Chicago, Illinois, USA, 1994.

19. El Ghaoui L, Niculescu S. Advances in Linear Matrix Inequality Methods in Control, Advances in Design and Control. SIAM: Philadelphia, PA, 2000.

20. Helton JW, McCullough S, Putinar M, Vinnikov V, Convex matrix inequalities versus linear matrix inequalities. IEEE Transactions on Automatic Control 2009; 54(5):952-964.

21. Boumans R, Heemskerk C. The European Robotic Arm for the international space station. Robotics and Autonomous Systems 1998; 23(1-2):17-27.

22. Yang Z, Blanke M. The robust control mixer module method for control reconfiguration. In Proceedings of American Control Conference 2000, Chicago, Illinois, USA, 2000; 3407-3411.

23. Yang Z, Blanke M, Verhaegen M. Robust control mixer method for reconfigurable control design using model matching. IET Control Theory and Applications 2007; 1(1):349-357.

24. Mattingley J, Wang Y, Boyd S. Receding horizon control. IEEE Control Systems Magazine 2011; 31(3):52-65.

25. Vargas AN, do Val JBR. Average cost and stability of time-varying linear systems. IEEE Transactions on Automatic Control 2010; 55:714-720. 